PRODUCTION

ENGINEERING

ARCHIVES
2015, No 4, pp 10-12

ISSN 2353-5156 (print version)

ISSN 2353-7779 (online version)

\title{
Analysis of impurity spaces as a percentage volume of non-metallic inclusions
}

\begin{abstract}
Tomasz Lipiński
University of Warmia and Mazury in Olsztyn, The Faculty of Technical Sciences, St: Oczapowskiego 11, 10-957 Olsztyn, Poland, +4889 5416191, tomek1@uwm.edu.pl

Abstract The parameters of high-grade steel are influenced by a combination of factors, including chemical composition and production technology. The impurity content is also a key determinant of the quality of high-grade steel. Non-metallic inclusions are one of the factors that influence the properties, in particular fatigue strength of steel. The physical and chemical reactions that occur in the process of steel melting and solidification produce non-metallic compounds and phases, referred to as inclusions. The quantity of non-metallic inclusions is correlated with the content of dopants in the alloy, while their phase composition and structure, in particular shape, dimensions and dispersion, impurity spaces are determined by the course of metallurgical processes. The experimental material consisted of semi-finished products of medium-carbon structural steel. The production process involved three melting technologies: steel melting in a basic arc furnace with: desulfurization or desulfurization and argon refining and in an oxygen converter and next subjected to vacuum circulation degassing. Billet samples were collected to analyze the content of non-metallic inclusions with the use of an optical microscope and a video inspection microscope. The application of various heat treatment parameters led to the formation of different microstructures responsible for steel hardness values. The objective of this study was to determine the influence of percentage volume non-metallic inclusions on impurity spaces.
\end{abstract}

Key words - steel, structural steel, non-metallic inclusions, oxideimpurities, fatigue strength, bending fatigue

\section{Introduction}

Although steel has a relatively small number of non-metallic inclusions, those impurities have a considerable impact on the material's technological and strength parameters, in particular fatigue strength and life. The composition of technical iron alloys is inclusive of sulfur and oxygen. Those elements form solutions in liquid metal. Then the presence of oxygen and non-metallic inclusions in steel is a natural consequence of physical and chemical processes during production. The shape of non-metallic inclusions may vary (BORKOWSKI S. 1999), (HONGAND T., DEBROY T. 2003), (UlEWICZ R. 2003), (MURAKAMI Y., ENDO M. 1994).
During processing, the shape and distribution of microparticles change, and impurities undergo anisotropic deformation. Non-metallic inclusions play a special role in the process of steel hardening. Due to differences in the physical properties of steel and inclusion-forming phases, structural stresses are formed along inclusion boundaries (CHICHKAREV E. A. 2009),(LIPIŃSKI T. WACH A. 2014, 2015), (WoŁCZYŃSKI W. 2015).

In a correctly performed metallurgical process, non-metallic inclusions in steel are randomly distributed, and their quantity can be described by the distances between the impurities of structural steel, which was investigated in the present study. 


\section{Aim of the study and methods}

The objective of this study was to determine changes in impurity space subject to percentage volume of inclusions of structural steel.

The tested material comprised steel manufactured in three different metallurgical processes. Steel was melted in basic arc furnace and desulfurized - A process, additionally refined with argon - B process. In the $\mathrm{C}$ process, steel was melted in oxygen converter and deoxidized by vacuum. Steel was cast continuously and square $100 \times 100 \mathrm{~mm}$ billets were rolled. Chemical composition was estimated with the use of quantometer, dimensions of impurities by inspecting metallographic specimens with the use of a video inspection microscope, determined for a larger boundary value of $2 \mu \mathrm{m}$.

Each of the heats $\lambda$ were calculated with the use of the below formula (1):

$\chi=\frac{2}{a} d\left(\frac{1}{w_{0}}-1\right)$

where: $V_{0}-$ the relative volume of impurities, $\%$,

$\bar{d}$ - the average diameter ofimpurity, $\mu \mathrm{m}$,

$\lambda$-arithmetic average impurities space.

The significance of correlation coefficients $r$ was determined on the basis of the critical value of the Student's t-distribution for a significance level $\alpha=0.05$.

\section{Results and discussions}

Statistically significant relationship impurity spaces of volume of non-metallic inclusions are described and applied to the regression equation in Fig. 1-9.

The impurities space $\lambda$ of steel melted in A process depending on percentage volume of inclusions are presented in Fig. 1.

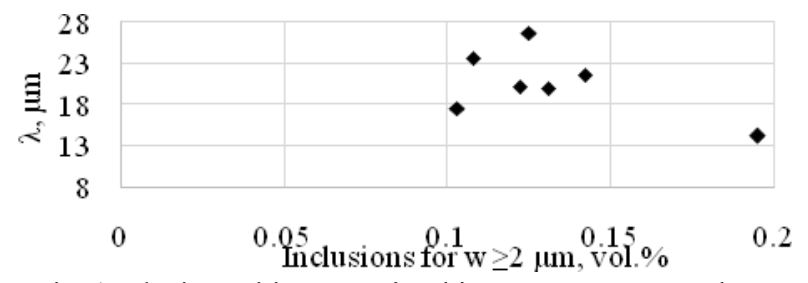

Fig. 1. The impurities space $\lambda$ subject to percentage volume of inclusions $\mathrm{w} \geq 2 \mu \mathrm{m}$ for A process.

The impurities space $\lambda$ of steel melted in B process depending on percentage volume of inclusions are presented in Fig. 2, regression equation and correlation coefficients $r$ at (2).

$$
\lambda=-97.515 \mathrm{~V}_{1}+23.611 \text { and } \mathrm{r}=0.8146
$$

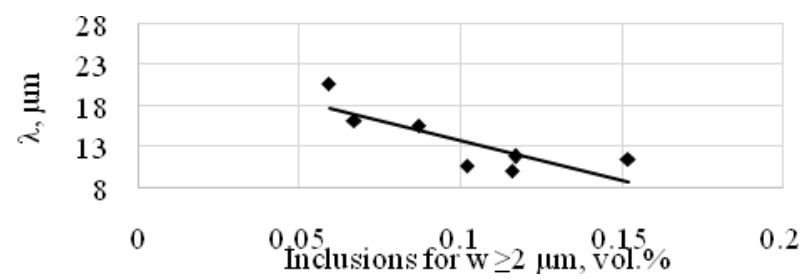

Fig. 2. The impurities space $\lambda$ subject to percentage volume of inclusions $\mathrm{w} \geq 2 \mu \mathrm{m}$ for $\mathrm{B}$ process.

The impurities space $\lambda$ of steel melted in C process depending on percentage volume of inclusions are presented in Fig. 3, regression equation and correlation coefficients $r$ at (3).

$\lambda=-718.44 \mathrm{~V}_{1}+55.752$ and $\mathrm{r}=0.7068$

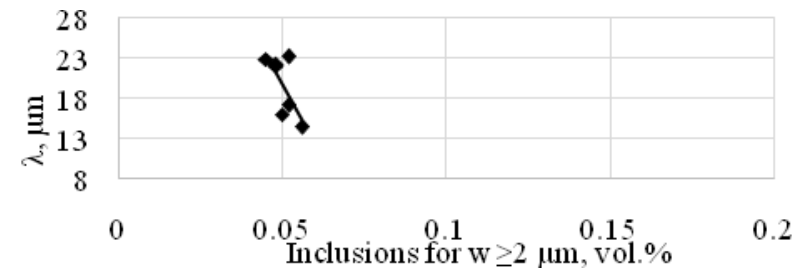

Fig. 3. The impurities space $\lambda$ subject to percentage volume of inclusions $\mathrm{w} \geq 2 \mu \mathrm{m}$ for $\mathrm{C}$ process.

The impurities space $\lambda$ of steel melted in A process depending on percentage volume of inclusionsare presented in Fig. 4.

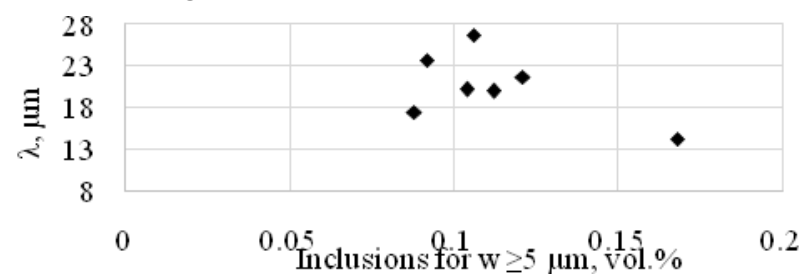

Fig. 4. The impurities space $\lambda$ subject to percentage volume of inclusions $\mathrm{w} \geq 5 \mu \mathrm{m}$ for A process.

The impurities space $\lambda$ of steel melted in B process depending on percentage volume of inclusions are presented in Fig. 5, regression equation and correlation coefficients $r$ at (4).

$\lambda=-123.72 \mathrm{~V}_{1}+23.705$ and $\mathrm{r}=0.7531$

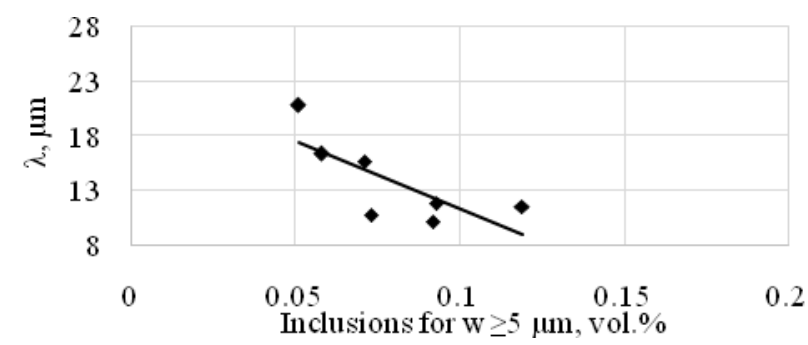

Fig. 5. The impurities space $\lambda$ subject to percentage volume of inclusions $\mathrm{w} \geq 5 \mu \mathrm{m}$ for B process. 
The impurities space $\lambda$ of steel melted in C process depending on percentage volume of inclusions are presented in Fig. 6.

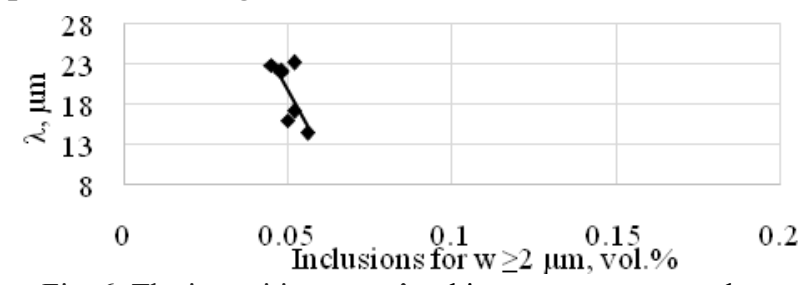

Fig. 6 . The impurities space $\lambda$ subject to percentage volume of inclusions $\mathrm{w} \geq 5 \mu \mathrm{m}$ for $\mathrm{C}$ process.

The impurities space $\lambda$ of steel melted in A process depending on percentage volume of inclusions are presented in Fig. 7, regression equation and correlation coefficients $r$ at (5).

$\lambda=-216.5 \mathrm{~V}_{1}+34.888$ and $\mathrm{r}=0.7601$

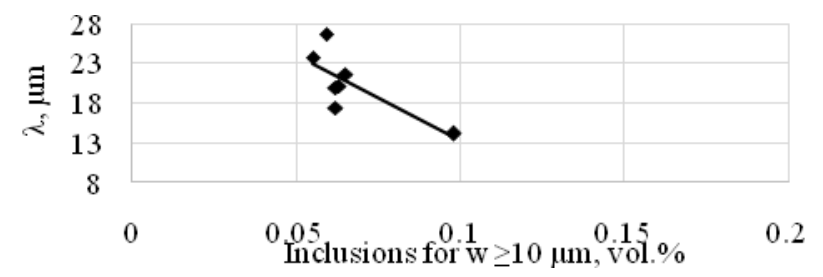

Fig. 7. The impurities space $\lambda$ subject to percentage volume of inclusions $\mathrm{w} \geq 10 \mu \mathrm{m}$ for A process.

The impurities space $\lambda$ of steel melted in B process depending on percentage volume of inclusions are presented in Fig. 8, regression equation and correlation coefficients $r$ at (6).

$\lambda=-214.65 \mathrm{~V}_{1}+23.795$ and $\mathrm{r}=0.7740$

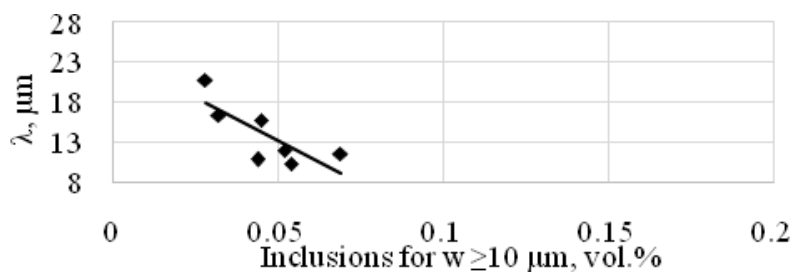

Fig. 8. The impurities space $\lambda$ subject to percentage volume of inclusions $\mathrm{w} \geq 10 \mu \mathrm{m}$ for $\mathrm{B}$ process.

The impurities space $\lambda$ of percentage volume of inclusions are presented in Fig. 9.

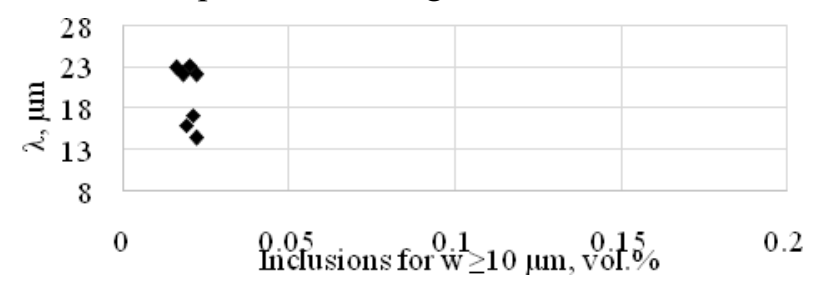

Fig. 9. The impurities space $\lambda$ subject to percentage volume of inclusions $\mathrm{w} \geq 10 \mu \mathrm{m}$ for $\mathrm{C}$ process.

\section{Summary and conclusions}

A method for melting in an electric furnace and subjected to desulfurization (A process)was a significant association only after reducing the differences in the size of the inclusions greater than $10 \mu \mathrm{m}$. Most likely, this can be explained by a high amount of impurities of different sizes and unsystematic distribution.

After the steel melt in $\mathrm{C}$ process, the amount of inclusions is small. Relevant equations were obtained only for the entire size range of the analyzed inclusions.

For process B regression equations are statistically significant for all analyzed ranges. This can be explained by the high effectiveness of out-of furnace treatment. As a result, a uniform distribution of impurities in individual intervals dimensional was obtained.

\section{Literature}

1. BORKOWSKI S.1999, Quality controlmaterialsforexampleironfoundry. WNT, Warsaw (in Polish).

2. ChichKarev E. A.2009, Conditions For Nonmetallic Inclusion Formation In Steels Deoxidized With Aluminum And Calcium. Metallurgist, 53, 728-734.

3. Hongand T., DeBroy T.2003, Nonisothermal growth and dissolution of inclusions in liquid steels. Metallurgical and Materials Transactions B 34B, 267-269.

4. LIPIŃSKI T., WACH A.2014, Influence of Outside Furnace Treatment on Purity Medium Carbon Steel. 23rd International Conference on Metallurgy and Materials METAL 2014. TANGER Ltd., Ostrava. Conference proceedings 738-743.

5. LIPIŃSKI T., WACH A.2015, Dimensional Structure of Non-Metallic Inclusions in High-Grade Medium Carbon Steel Melted in an Electric Furnace and Subjected to Desulfurization. Solid State Phenom.223, 46-53.

6. ULEWICZ R.2003, Quality control system in production of the castings from spheroid cast iron. 42 (1), 61-63.

7. MURAKAMI Y., ENDO M. 1994, Effects of defects, inclusions and in homogeneities on fatigue strength, International Journal of Fatigue 16(3), 163-82.

8. WotCZYŃSKI W.2015, Mathematical Modeling of the Microstructure of Large Steel Ingots,in: The Encyclopedia of Iron, Steel, and Their Alloys, Eds. Taylor \& Francis Group, New York-USA, (in print). 